Le temps des grands festins de Noël est fini, arrive celui des bonnes résolutions; peut-être la lecture du sixième rapport sur la nutrition en Suisse vous aidera-t-elle à les suivre. La Suisse ne fait pas exception en ce qui concerne le surpoids, avec les conséquences que l'on connaît pour la santé. Pourquoi avonsnous donc tant de peine à mettre en pratique les bons réflexes alimentaires qui nous sont pourtant connus? Jetons un coup d'œil en arrière: durant plusieurs centaines de milliers d'années, il était très important de manger beaucoup pour survivre, lorsque la nourriture était disponible, et de constituer des réserves de graisse pour les périodes maigres. Nous bougions sans cesse; se procurer de la nourriture était une entreprise qui demandait des efforts considérables! Nous ne sommes pas faits pour l'opulence et des rayons de vente débordants. Nous devons joindre nos efforts pour que nos instincts hérités de nos ancêtres vivant de la chasse et de la cueillette, ne nous entraînent dans un déséquilibre dangereux en raison de la surabondance de nourriture et du manque d'activité physique.

\title{
Sixième rapport sur la nutrition en Suisse et Stratégie alimentaire suisse 2013-2016
}

\author{
Andrea Renggli, Michael Beer, \\ Pascale Mühlemann, \\ Nadine Stoffel-Kurt
}

Correspondance: Andrea Renggli

Office fédéral de la santé publique,

Division Sécurité alimentaire, section Risques nutritionnels et toxicologiques

Stauffacherstrasse 101

CH-8004 Zurich

andrea.renggli[at]bag.admin.ch
Santé et alimentation sont étroitement liées: équilibrée, l'alimentation couvre les besoins en énergie (calories) et en nutriments, permet d'éviter un apport énergétique excessif et influe sur le bien-être physique et mental. De plus, elle contribue à conserver la santé et à prévenir les maladies liées à la nutrition. Selon l'Organisation mondiale de la santé (OMS), quatre des principaux facteurs favorisant l'apparition de maladies non transmissibles sont liés à l'alimentation: consommation insuffisante de fruits et de légumes, hypertension, surpoids et obésité ainsi que taux de cholestérol trop élevé.

Les rapports sur la nutrition en Suisse publiés tous les sept ans par l'Office fédéral de la santé publique (OFSP) constituent un instrument important pour rendre compte de la situation. Ils s'adressent aussi bien aux spécialistes qu'à la population intéressée. Le $6^{\text {e }}$ RNS, de 300 pages environ, que le conseiller fédéral Alain Berset a présenté le 22 janvier 2013, comporte quatre chapitres:

- Recommandations alimentaires actuelles

- Situation nutritionnelle en Suisse

- Alimentation et santé

- Mesures nutritionnelles destinées à promouvoir la santé

\section{Recommandations alimentaires}

Les recommandations alimentaires en Suisse s'adressent à différentes tranches d'âge et à des groupes de population spécifiques. Elles se basent sur les principes de l'OMS et ont été adaptées à notre pays par différentes institutions et associations professionnelles. Il s'est avéré que, pour préserver la santé et prévenir les maladies, il était important de disposer aussi bien de recommandations quantita-
Le sixième rapport sur la nutrition en Suisse (6 ${ }^{\mathrm{e} N S}$ ), qui vient d'être publié, décrit la situation actuelle et présente les mesures aptes à l'améliorer. Au cours des trois dernières décennies, I'utilisation de denrées alimentaires (kg par habitant) n'a pas sensiblement évolué. Le nombre important de personnes en surpoids et obèses constitue un des défis majeurs. En Suisse, beaucoup de programmes et de projets visent à promouvoir une alimentation équilibrée et l'activité physique; un bon nombre d'entre eux agissent aussi bien sur le comportement que sur le contexte de vie. Sur la base des conclusions du $6^{\mathrm{e}}$ RNS, six domaines d'action ont été formulés dans la Stratégie alimentaire suisse 2013 2016; ils définissent les objectifs et les priorités dans le domaine de la nutrition.

tives (par ex., «des fruits et des légumes cinq fois par jour»), que qualitatives (choix de l'huile selon sa composition en acides gras). La version actuelle de la pyramide alimentaire suisse, que la Société suisse de nutrition (SSN) et l'OFSP ont publiée ensemble en décembre 2011, indique les aliments qui devraient être moins consommés et ceux qui devraient l'être plus. Beaucoup de personnes connaissent les recommandations alimentaires les plus importantes mais, pour différentes raisons, ne les appliquent pas ou partiellement. Près de $30 \%$ de la population ne porte aucune attention particulière à son choix nutritionnel.

Certaines recommandations alimentaires peuvent présenter des contradictions. Ainsi, les poissons de mer gras, par exemple, constituent une bonne source 


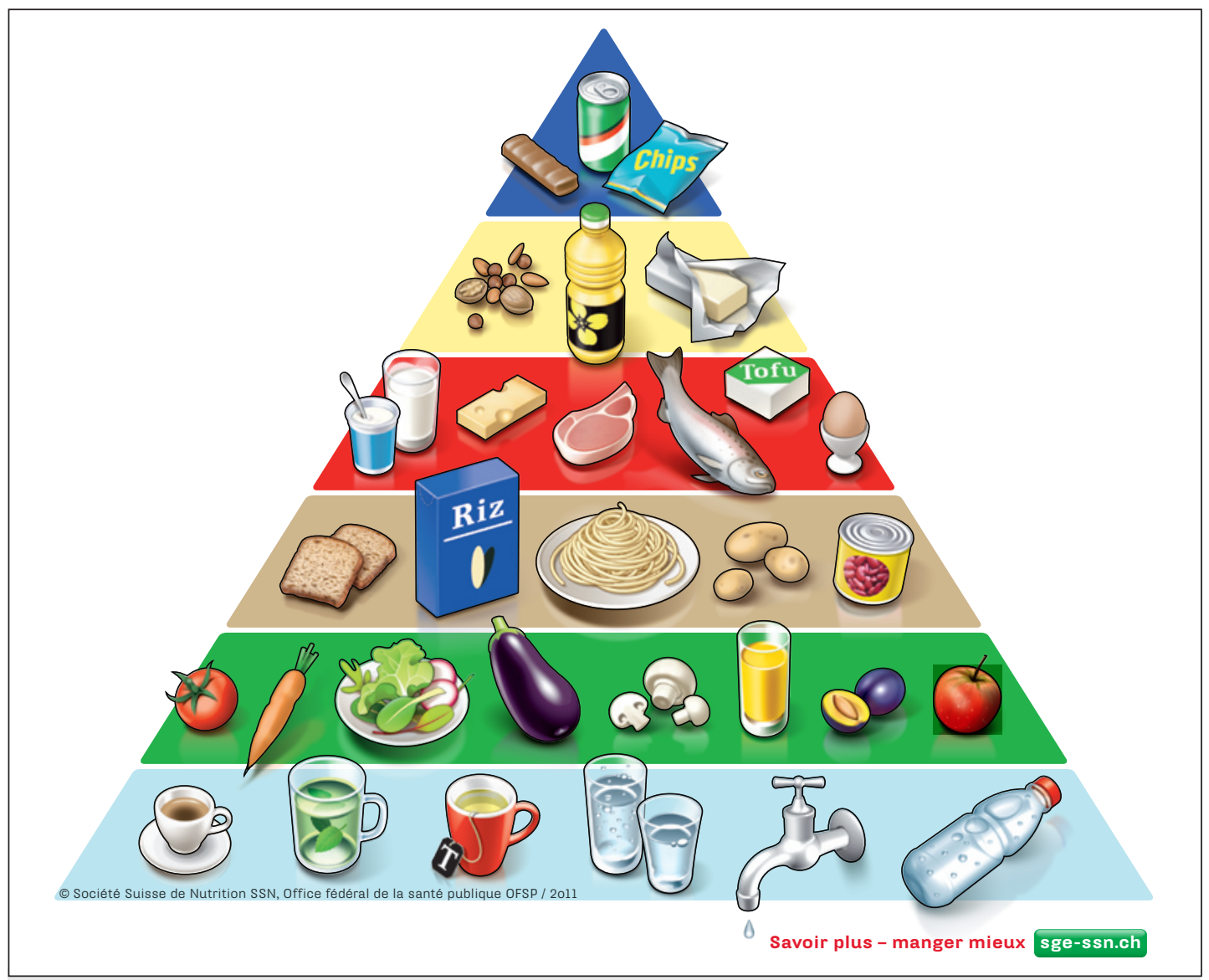

Beaucoup de personnes connaissent les recommandations alimentaires les plus importantes mais, pour différentes raisons, ne les appliquent pas ou partiellement.

en acides gras poly-insaturés à longue chaîne, mais ils peuvent contenir des métaux lourds. Afin d'éviter que les recommandations ne présentent une menace pour la santé, une analyse soigneuse des avantages et des risques s'impose.

Outre l'alimentation, le 6e RNS établit des recommandations basées sur les nutriments. L'accent est en particulier mis sur le sodium, le sel et la vitamine $\mathrm{D}$, notamment suite à la «Stratégie sel» de l'OFSP et à la discussion sur les besoins et l'apport de vitamine D en Suisse.

\section{Utilisation de denrées alimentaires et apport en nutriments}

La Suisse ne dispose pas de données alimentaires individuelles. Les informations sur l'utilisation qui figurent dans le $6^{\text {e }}$ RNS se basent sur les statistiques agricoles et correspondent aux quantités d'aliments disponibles sur le marché. Cette lacune, mentionnée à plusieurs reprises dans le $6^{\mathrm{e}} \mathrm{RNS}$, peut maintenant être comblée grâce à l'étude nationale sur la nutrition, financée par l'OFSP, et lancée cette année.

$\mathrm{Au}$ cours des trois dernières décennies, l'utilisation de denrées alimentaires (kg par habitant) par la population suisse n'a pas sensiblement évolué. Sur la base des données approximatives concernant la consommation alimentaire, il semble que la plupart des micronutriments soient absorbés en quantités suffisantes, à l'exception de l'acide folique et de la vitamine D. En ce qui concerne le fer, la vitamine B1 et le magnésium, les quantités sont tout juste suffisantes. Vu l'absence de données (par ex., prises de sang), il n'est pas possible de fournir des indications plus précises sur l'importance et la prévalence ou non d'une carence en micronutriments. Il semble toutefois que l'approvisionnement de la population, ou du moins d'une partie d'entre elle, en vitamine $\mathrm{D}$, en acide folique, en iode et probablement aussi en fer, soit insuffisant. Le sélénium, par contre, est consommé en quantités suffisantes.

Aujourd'hui déjà, environ une personne hospitalisée sur quatre ou sur cinq présente des carences alimentaires ou risque d'en présenter. Cette problématique devrait prendre de l'importance au cours des prochaines années avec l'augmentation du nombre de personnes âgées.

\section{Alimentation et santé}

Le nombre d'adultes atteints de surpoids et d'obésité n'a cessé d'augmenter en Suisse au cours des 30 dernières années. Les hommes sont nettement plus touchés par le surpoids (indice de masse corporelle, 
IMC $\left.=25,0-29,9 \mathrm{~kg} / \mathrm{m}^{2}\right)$ que les femmes $(37,6 \%$ contre $20,8 \%$ ). En ce qui concerne l'obésité (IMC $\geq$ $\left.30 \mathrm{~kg} / \mathrm{m}^{2}\right)$, la différence entre les sexes est nettement moins marquée ( $8,7 \%$ pour les hommes et $7,8 \%$ pour les femmes). Chez les enfants, la proportion varie suivant l'âge et l'étude considérée: entre 5 à $20 \%$ de surpoids et 0,4 à $6 \%$ d'obésité. Il semble que chez les adultes comme chez les enfants, le surpoids et l'obésité se stabilisent, mais à un niveau élevé.

Bon nombre de maladies liées à la nutrition comme l'hypertension, le diabète sucré de type 2 , les maladies cardio-vasculaires ainsi que certaines formes de cancer sont liées au surpoids et à l'obésité. Bien que ces deux phénomènes aient engendré des coûts directs et indirects de 5,8 millions de francs en 2006, seuls 2,3\% des dépenses du secteur de la santé sont affectées à la prévention et à la promotion de la santé. Dans les pays de l'OECD, la moyenne atteint $3,1 \%$. fants et optimiser la composition des offres de repas pris à l'extérieur. La responsabilité d'une mise en œuvre fructueuse des mesures incombe, entre autres, aux différentes autorités politiques, aux écoles, aux organismes de formation, aux organisations non gouvernementales et à l'économie privée (par ex., l'industrie agroalimentaire, la gastronomie).

\section{La Stratégie alimentaire suisse 2013-2016}

L'analyse de la situation établie dans le $6^{\text {e }}$ RNS permet d'identifier les problèmes nutritionnels. On peut ainsi définir des domaines d'action à l'intérieur desquels il sera possible de prendre des mesures concrètes visant à améliorer la situation nutritionnelle et sanitaire. La publication du $6^{e}$ RNS a été l'occasion, pour l'OFSP, de remanier la stratégie de 2001. Parallèlement au 6e RNS, il a aussi publié la Stratégie alimentaire suisse 2013-2016. Cette stratégie, adoptée par le département, détermine les priorités et les

\section{«Aujourd'hui déjà, environ une personne hospitalisée sur quatre ou sur cinq présente des carences alimentaires ou risque d'en présenter.»}

\section{Activités en cours, groupes cibles et actions recommandées}

En Suisse, de nombreux programmes et projets visent à promouvoir une alimentation équilibrée et l'activité physique. Les mesures qui tiennent compte de l'environnement et du cadre de vie du groupe cible, et qui agissent donc aux niveaux comportemental et contextuel, se sont avérées prometteuses. A l'avenir, les interventions concernant la nutrition devraient en particulier viser les hommes, les jeunes et la population défavorisée du point de vue socioéconomique. L'accent devrait surtout être mis sur les mesures contextuelles.

Le $6^{\mathrm{e}}$ RNS présente des actions recommandées et des mesures urgentes pour la Suisse. Ces dernières consistent, par exemple, à: améliorer les données à disposition, renforcer les compétences personnelles en matière de santé et de nutrition, promouvoir la consommation de fruits et de légumes chez les en- objectifs et offre simultanément une base aux différents acteurs pour l'élaboration de leurs propres plans d'action et mesures. La Stratégie alimentaire suisse constitue par ailleurs la base centrale du Programme national alimentation et activité physique (PNAAP), que le Conseil fédéral a prolongé, en été 2012, jusqu'à fin 2016. Pour que le projet réussisse, il faut, d'une part, bien coordonner les interventions aux niveaux comportemental et contextuel et, d'autre part, faire coopérer les acteurs de la nutrition et de la santé, mais aussi de l'économie, de la formation et de l'agriculture.

Le $6^{\text {e }}$ RNS et la Stratégie alimentaire suisse 20132016 (brochure) sont proposés à titre gratuit en allemand, en français et en italien sous forme électronique à l'adresse:

- www.ernaehrungsbericht.ch

- www.rapportsurlanutrition.ch

- www.rapportosullalimentazione.ch 